09.2

\title{
Пространственные оптические ловушки на основе многопучковой интерференции
}

\author{
() Н.В. Шостка (Ляхович), Б.В. Соколенко, О.С. Каракчиева (Сидоренкова), В.И. Шостка \\ Крымский федеральный университет им. В.И. Вернадского, Симферополь, Россия \\ E-mail: nataliya_shostka@mail.ru
}

Поступило в Редакцию 9 апреля 2020 г.

В окончательной редакции 9 апреля 2020 г.

Принято к публикации 29 июля 2020 г.

\begin{abstract}
Представлена модель формирования пространственных оптических ловушек для захвата, перемещения и угловой ориентации микрочастиц на основе суперпозиции нескольких гауссовых пучков в различной геометрической конфигурации с управляемыми параметрами.
\end{abstract}

Ключевые слова: оптическая ловушка, интерференция, гауссов пучок.

DOI: 10.21883/PJTF.2020.22.50299.18332

Контролируемый захват микрообъектов в различных средах, а также манипулирование их пространственным положением вдоль заданных траекторий с помощью света является одним из актуальных приложений лазеров с момента их появления. Актуальной задачей современных исследований является дальнейшее усовершенствование метода оптического пинцета и различных его конфигураций как прекрасного прикладного высокоточного инструмента в биологии и медицине для осуществления неинвазивного пространственного позиционирования биологических объектов в естественной среде. Для большинства современных прикладных задач реализации оптического захвата используются трехмерные оптические ловушки, представляющие собой равномерно окруженную максимумами интенсивности область с минимальной интенсивностью света $[1,2]$. Такие оптические структуры могут быть получены как в когерентном, так и в частично когерентном свете, например, при помощи аксикона [3,4].

Одним из знаковых моментов, повлиявших на развитие современной оптики, в том числе и направления реализации оптических ловушек, является создание пространственных световых модуляторов (SLM), позволяющих достаточно просто и точно формировать различные оптические пучки, а также их массивы, свойства которых можно изменять независимо и в режиме реального времени $[5,6]$. В $[7,8]$ проанализирован процесс параметризации ловушки с помощью реконфигурируемых массивов пучков высшего порядка, включающих как скалярные сингулярные пучки, так и векторные с радиальным и азимутальным распределениями поляризации в одной и той же конфигурации системы ловушек.

Цель данного исследования состоит в разработке метода синтеза пространственных оптических ловушек за счет интерференционных эффектов определенного числа (от трех до пяти) лазерных пучков, управляющими параметрами суперпозиции которых являются углы наклона и прецессии относительно оси распространения, а также линейный сдвиг в поперечной плоскости от данной оси.

Отметим, что формирование тонкой интерференционной двумерной структуры на основе трех гауссовых пучков встречается в исследованиях $[9,10]$, направленных на решение прикладных задач микро- и нанолитографии, в фотонных кристаллических устройствах [11] и микроскопии [12]. В качестве пространственной трехмерной структуры для захвата и удержания микрообъектов данные решения рассматривались частично в [2], а анализ пространственной эволюции поля в продольной и поперечной плоскости ранее не проводился.

Рассмотрим распространение монохроматического наклонного гауссова пучка с длиной волны $\lambda$ под некоторым малым углом $\alpha_{0}$ к оси $z$ (рис. 1,a), при этом мы ограничимся параксиальным приближением, что накладывает условие вида $\sin \alpha_{0} \ll 1$, так что $\cos \alpha_{0} \approx 1-\alpha_{0}^{2} / 2$. Напряженность электрического поля пучка будем описывать выражением

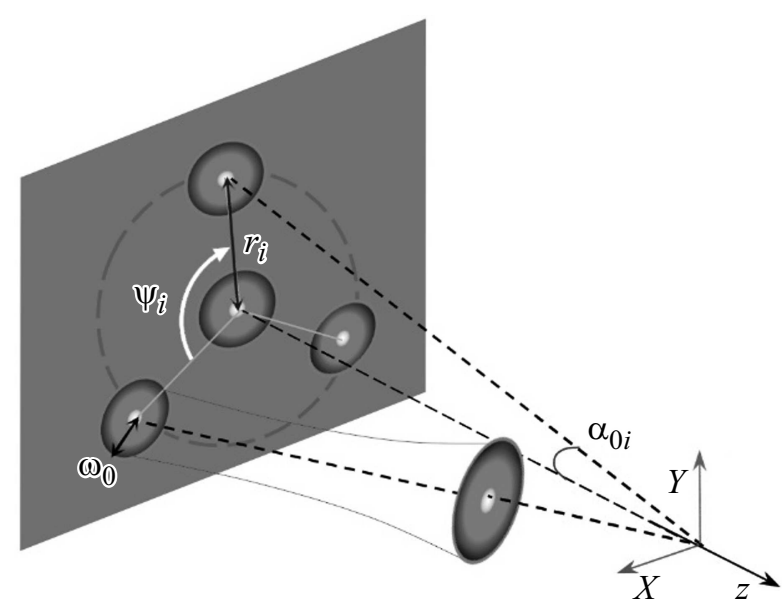

Рис. 1. Геометрия распространения интерферирующих пучков в пространстве для случая четырех пучков, сходящихся под углом $\alpha_{0}$ к оси распространения $z$. 

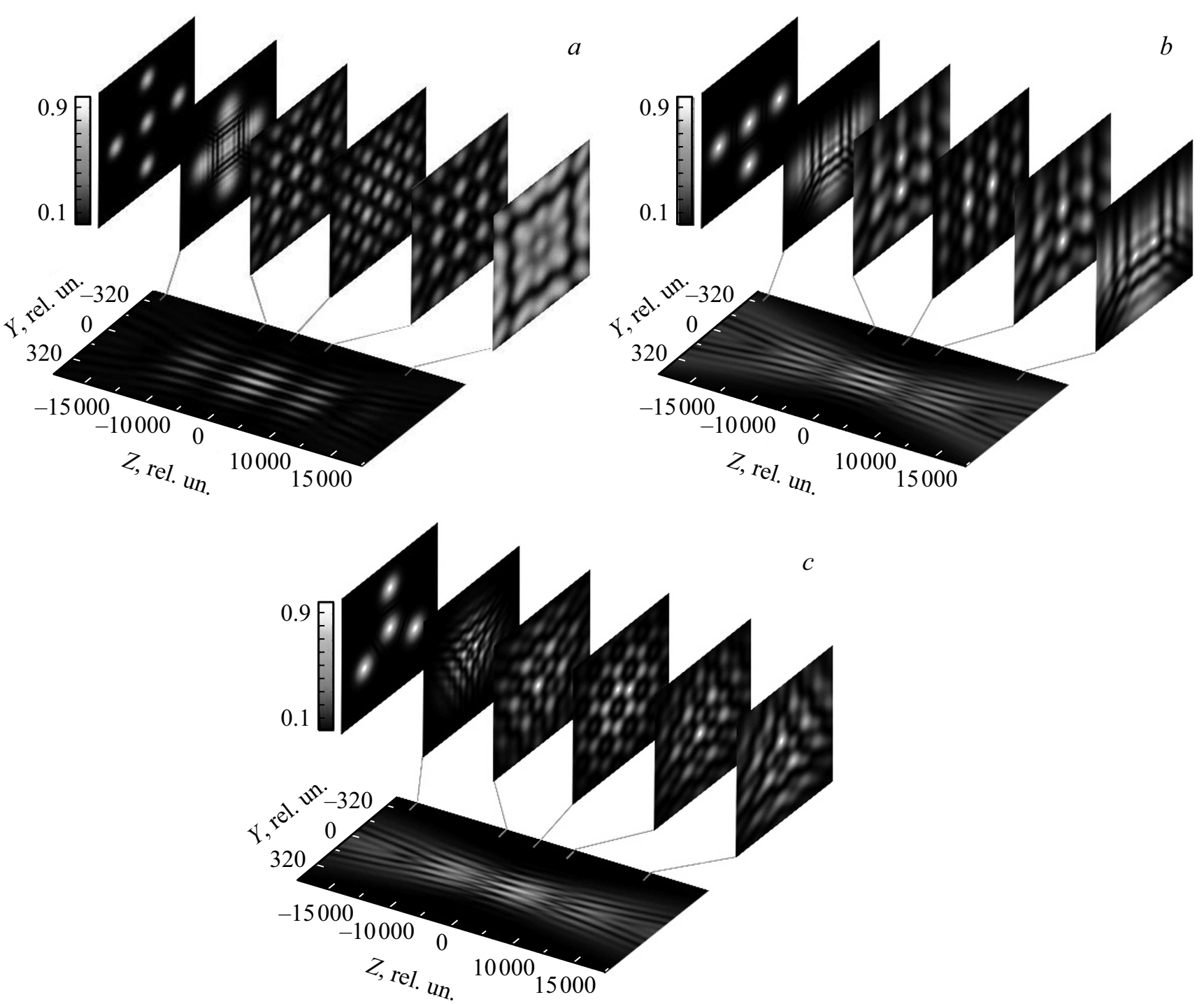

Рис. 2. Рассчитанные интерференционные картины суперпозиции пяти $(a)$ и четырех $(b, c)$ пучков с различной конфигурацией взаимного расположения, формирующие пространственные ловушки „закрытого“ типа за счет центрального осевого пучка.

$E=\tilde{E}(x, y, z) \exp (-i k z+i \varphi)$, где $\varphi-$ начальная фаза, $k=2 \pi / \lambda-$ волновое число, $\tilde{E}-$ комплексная амплитуда, удовлетворяющая уравнению Гельмгольца

$$
\partial_{x}^{2} \tilde{E}+\partial_{y}^{2} \tilde{E}-2 i k \partial_{z} \tilde{E}=0 .
$$

Простейшим решением данного уравнения является параксиальный гауссов пучок, в рассматриваемом случае распространяющийся наклонно по отношению к оси $z$ и характеризующийся преобразованием координат в плоскости $x 0 z$ вида $x \rightarrow X=x+i \alpha_{0 i} z_{0}$, а в плоскости $y 0 z-$ вида $y \rightarrow Y=y+i \alpha_{0 i} z_{0}$, где $z_{0}=k_{0} \omega_{0}^{2} / 2, \omega_{0}-$ радиус перетяжки пучка в плоскости $z=0$ [13]. С учетом всех параметров (угла прецессии $\psi_{i}-$ поворота вокруг оси $z$ - и смещения центра пучка в поперечной плоскости $z=0$ от оси на величину $r_{i}$ ) окончательный вид преобразования координат запишется в виде

$$
\begin{gathered}
X=\left(i \alpha_{0 i} z_{0}+x\right) \cos \psi_{i}+\left(y+r_{i}\right) \sin \psi_{i}, \\
Y=\left(i \alpha_{0 i} z_{0}-x+r_{i}\right) \sin \psi_{i}+y \cos \psi_{i} .
\end{gathered}
$$

Решением уравнения (1) является комплексная амплитуда с гауссовой образующей

$$
\begin{gathered}
\tilde{E}_{i}(X, Y, z)=\frac{1}{1-i z / z_{0}} \exp \left[-\frac{X^{2}+Y^{2}}{\omega_{0}^{2}\left(1-i z / z_{0}\right)}\right] \\
\times \exp \left(-\alpha_{0 i}^{2} k z_{0}\right) .
\end{gathered}
$$

По определению распределение интенсивности поля интерферирующих волн описывается выражением [10]:

$$
I(X, Y, z)=\sum_{i=1}^{N}\left|E_{i}\right|^{2}+\sum_{i \neq j}^{N} E_{i} E_{j},
$$

представляющим собой сумму квадратов амплитуд и интерференционные члены пар пучков с соответствующими индексами $i, j$, которые изменяются в нашем случае в диапазоне от 1 до $N$, где $N$ - число интерферирующих пучков, амплитуда которых определяется 

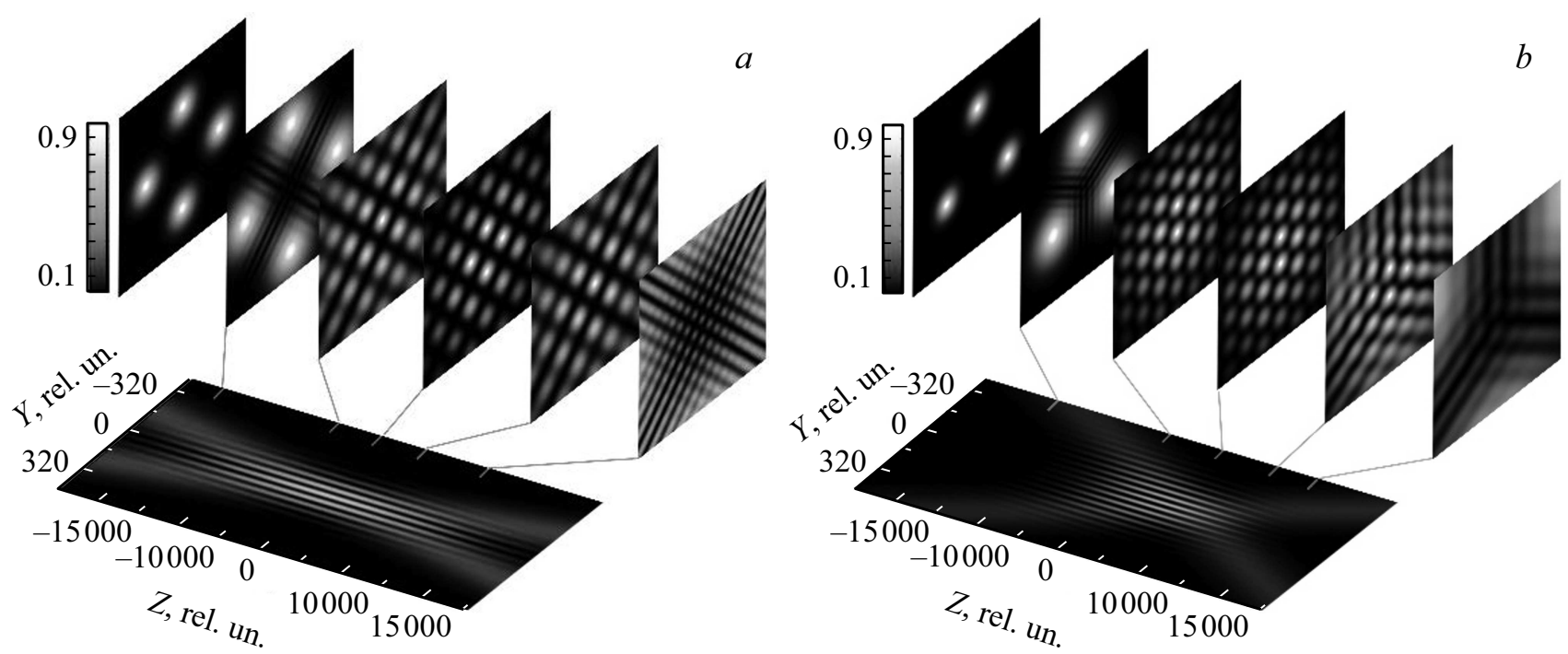

Рис. 3. Рассчитанные интерференционные картины суперпозиции четырех $(a)$ и трех $(b)$ пучков, формирующие пространственные ловушки „открытого“ типа.

выражением (2) и в простейшем случае может быть одинаковой для всех пучков. Таким образом, геометрическая пространственная структура интерференционного поля будет описываться соответствующим набором параметров $\psi_{i}, r_{i}$ и $\alpha_{0 i}$ отдельного пучка. На рис. 2 представлены результаты численного моделирования распространения $N$ пучков различной конфигурации с осевым центральным пучком и соответствующая им динамика интерференционной картины. Масштаб приведен в относительных единицах $z / \lambda, Y / \lambda$.

Характер формирования интерференционных минимумов и максимумов в виде структуры ячеек на рис. 2, $a$ обусловлен одинаковым углом наклона пучков по отношению к центральному пучку на угол $\alpha_{0 i}=0.03 \mathrm{rad}$ и равным расстоянием от его оси. Такое расположение пучков дает равномерную локализацию максимумов и минимумов интенсивностей как в поперечной, так и в продольной плоскости, расстояние между ближайшими максимумами равно $d=\lambda /\left(\sqrt{2} \sin \alpha_{0 i}\right)$ [11]. Для случая, изображенного на рис. $2, b$, несимметричное расположение пучков обусловливает снижение контраста и деформацию структуры максимумов.

Картина заметно изменяется при симметричном расположении трех периферических пучков относительно центрального на одинаковом расстоянии $r_{i}$, как показано на рис. 2,c. В данном случае центральный пучок позволяет сформировать ловушки в ,закрытом“ состоянии: каждый минимум интенсивности окружен соседними максимумами, расположенными в продольной и поперечной плоскостях. Такие локальные структуры известны также как „бутылочные“ пучки [14]. При этом в поперечной плоскости формируется гексагональная структура с ярко выраженным центральным минимумом. Полученная симметрия поля пучка может быть использована для захвата поглощающих свет частиц, размеры которых превышают $d$, в центральную область с минимумом интенсивности, а более мелких объектов с высоким показателем преломления - в ячейки с максимумом интенсивности.

Отдельно следует отметить генерацию „открытых“ ловушек при отсутствии осевого пучка (рис. 3, $a, b$ ). Тонкая периодическая структура в продольном направлении разрушается, тем не менее в поперечном сечении пучков наблюдаются ячейки с ярко выраженными максимумами интенсивности округлой формы, в свою очередь являющиеся „открытыми“ в продольном направлении. Такая геометрия суперпозиции пучков может быть использована для двумерного захвата и поворота массива объектов с показателем преломления больше показателя окружающей среды.

Полученные результаты демонстрируют возможность применения суперпозиции трех и более пучков для генерации пространственных оптических ловушек „бутылочных“ пучков. Управление расположением ловушек в виде квазипериодической структуры минимумов и максимумов интенсивности обеспечивается изменением параметров конфигурации интерферирующих пучков: угла наклона пучков относительно оси распространения, их взаимного расположения и количества. Такой подход позволяет создать простой механизм реализации оптических манипуляторов на практике с применением пространственных световых модуляторов, амплитудных экранов и интерферометров на основе деления волнового фронта.

\section{Финансирование работы}

Работа выполнена при поддержке Российского фонда фундаментальных исследований и Совета министров Республики Крым (грант № 19-42-910010 p_a), а также стипендии Президента РФ молодым ученым № СП-745.2019.4. 


\section{Конфликт интересов}

Авторы заявляют, что у них нет конфликта интересов.

\section{Список литературы}

[1] Arlt J., Padgett M.J. // Opt. Lett. 2000. V. 25. N 4. P. 191-193. DOI: $10.1364 /$ OL.25.000191

[2] Shvedov V.G., Hnatovsky K., Shostka N.V., Rode A., Krolikowsky W. // Opt. Lett. 2012. V. 37. N 11. P. 19341936. DOI: $10.1364 /$ OL.37.001934

[3] Yang Z., Lin X., Zhang H., Xu Y., Jin L., Zou Y., Ma X. // Opt. Lasers Eng. 2020. V. 126. P. 105899.

DOI: 10.1016/j.optlaseng.2019.105899

[4] Yang Z., Lin X., Zhang H., Ma X., Zou Y., Xu L., Xu Y., Jin L. // Appl. Opt. 2019. V. 58. N 10. P. 2471-2480. DOI: $10.1364 / A O .58 .002471$

[5] Porfirev A.P., Dubman A.B., Porfiriev D.P. // Opt. Lett. 2020. V. 45. N 6. P. 1475-1478. DOI: 10.1364/OL.386907

[6] Ghebjagh S.G., Fischer D., Sinzinger S. // Appl. Opt. 2019. V. 58. N 32. P. 8943-8949. DOI: 10.1364/AO.58.008943

[7] Bhebhe N., Williams P., Rosales-Guzmán C., RodriguezFajardo V., Forbes A. // Sci. Rep. 2018. V. 8. P. 17387. DOI: 10.1038/s41598-018-35889-0

[8] Gong Z., Pan Y., Videen G., Wang C. // J. Quant. Spectrosc. Radiat. Transf. 2018. V. 214. P. 94-119. DOI: $10.1016 /$ j.jqsrt.2018.04.027

[9] Beckemper S., Huang J., Gillner A.D., Wang K. // JLMN. 2011. V. 6. N 1. P. 49-53. DOI: 10.2961/jlmn.2011.01.0011

[10] Ionel L., Zamfirescu M. // Romanian Rep. Phys. 2017. V. 69. N 1. P. 402.

[11] Burrow G.M., Gaylord T.K. // Micromachines. 2011. V. 2. N 2. P. 221-257. DOI: $10.3390 / \mathrm{mi} 2020221$

[12] Senthilkumaran P., Masajada J., Sato S. // Int. J. Opt. 2012. V. 18. P. 517591. DOI: 10.1155/2012/517591

[13] Fadeyeva T.A., Rubass A.F., Sokolenko B.V., Volyar A.V. // J. Opt. A. 2009. V. 11. N 9. P. 094008. DOI: 10.1088/1464-4258/11/9/094008

[14] Shostka N.V., Karakchieva O.S., Sokolenko B.V., Shostka V.I. // J. Phys.: Conf. Ser. 2019. V. 1400. P. 066028. DOI: $10.1088 / 1742-6596 / 1400 / 6 / 066028$ 